\title{
Using the Cloud-based Creative Services to Study and Design Q-Version Comic System
}

\author{
Shang-Te Tsai ${ }^{1 *}$, Guo-Bin You ${ }^{1}$, Cheng-Fu Yang ${ }^{2}$ \\ ${ }^{1}$ Department of School of Economics and Management, Ningde Normal University, Ningde City, \\ Fujian Province Economic Development Zone, Xueyuan Road City, No.1, corresponding author: feilongzz@gmail.com \\ ${ }^{2}$ Department of Chemical and Materials Engineering, National University of Kaohsiung, Kaohsiung 811, Taiwan
}

\begin{abstract}
The cultural creative industry has grown rapidly all over the world recently. By using cloud computing technologies, comic designers can publish their product easily through Internet. However, the novice comic creators are suffered developing comic characters and its style. To solve this problem, this study provides comic designer with service mechanism based on cloud computing to generate character style easily by applying rough set and grey prediction so that novice can create different character in certain context automatically, and contribute to cultural creative industry. The online style generating systems was implemented and tested to achieve this goal.
\end{abstract}

Key words: Cloud-based, Creative Services, Q-Version Comic, Design Element

\section{Introduction}

\subsection{Cultural Creative Industry Development}

The rapid development of Internet technology enables a lot of opportunities of systems developer to provide innovative online services and contribute to business. One of these promised technologies is cloud computing [3] which can solve many problems caused by limited budget and lack of expertise to deploy enterprise systems and online services. Creative Services means that a company can provide necessary support on the services of creativity to other companies or to the public. Typical business models revolve at the selling time of skilled professionals, either based on a project-by-project basis, or through an interacting contract and providing services on an ongoing basis as the companies pay a fixed monthly fee. Many company constructed the Creative Services [10] on the cloud network for that the people can obtain and use these software and services easily.

In Taiwan, government announced that cultural creative industry will be the key economic development policy in 2008 . Moreover, the other economic policy followed by cultural creative industry was cloud computing which is included in "Four Intelligent Emerging Industries" and was announced in 2010. These polices encourage enterprises and industry to develop online platform and mechanisms to support collaborate design[12] and more creative con-tents can be produced online. This study intend to bridge the gap between cultural creative industry and the ICT industry by support content creators with services to collaborate[6] their design online and to fulfill the need of future trend.

1.2 Online Style Generating Systems

In the past, $\mathrm{Qu}$ et al presented a discrete event simulation model (DESM) to substitute for the traditional method for making a novel prediction and schedule producing system [8]. Through the extension, the DESM is able to be started from any initial state. They also proposed a novel schedule generating system through combining the proper analysis of search algorithm with the prediction of using DESM. Creating website for special purpose has become more and more complex and it is usually not a job which can be finished by one person. It is important to confirm that the design is consistent and optimized to meet the requirements of business objectives and can create enjoyable experiences for different users.

One of the ways to confirm that team is working on the same page when they are arranged to design separate parts of the website or save designs from developers is to create design documentation or a web design style guide. For that, this study applies cloud computing architectural to develop online style generating systems (OSG systems) which support content creators to collabo-rate their cultural content design. The OSG system is based on "online Style database" to accelerate de-signer's design process by providing them an online automatic matching service to accomplish comic character style design. The mechanisms of these services are depends on data mining technics to provide most fit character style design according to the "main creative elements" and "minor creative elements". This online service can help character de-signer with less effort to try out character style design properly and less time consuming.

\subsection{The Architectural of OSG Systems}

Q-version is the English translation for the Chinese words, it means that the images, materials, or animals look so cute and funny. The implementation of OSG systems is designed in a Q-version comic context. Each character is combined [1] by different parts which are already created by designer and are categorized and uploaded into online style database. The "main creative elements [2]" is key attribute and is defined to Q-version face elements. The OSG systems will then rely on main creative elements and minor creative elements and using gray relational mechanisms to generate proper character style [4].

\section{Authoring System Based On Cloud Sharing}

Cloud is an easily obtainable source that people can use the software to calculate, simulate, or design many data and these can also be stored in the cloud system. Computing data in a cloud system has become a concept and a buzzing word in the necessary computing system for many years, this technology has been widely used with many businesses. But it is not fully understand what it actually is and how it will have a benefit 
from this system [9]. Computing in the cloud system typically consists of the software transfer services, computing ability, and data storage on the internet system. Cloud computing has above outlined characteristics through three main services that have come to define cloud computing and how the end users can enjoy and use these resources and services. An important benefit of using cloud computing is that it can be infinite on demand scalability, which personal computers can't easily and readily provide. Cloud-based content is eLearning authoring and it can be free from the constraints of typical personal computers' solutions. Users' access authoring software over the Internet via a secure identification, this method has a benefit of affordable hosted system - users do not need worrying about software set-up. Visual design elements and principles have described fundamental ideas about the practices of good visual designs. It means that the design elements are the basic units of any visual designs, which can form its structure and convey the necessary visual messages. Creative elements can be used to specialize in the productions of films, music videos, photography, web videos, and many other forms of media contents. The main creative elements in OSG systems is de-fined as "face", minor creative elements includes "body", "background", "item", and "other". By applying OSG systems in the cloud, output will be different "creator" in a certain context, as the structure shown in Figure 1. We can obtain many shared authoring system design architecture from the cloud-system, for example, the different personages, different action, and different dancing style. To enrich the online style database [7], each individual designer is allowed to upload his/her "main creative elements" or "minor creative elements", so every designer who joined this platform is free to access online style database. Figure 2, and more creative character will be made therefore, as the obtained data from the cloud and combined the "main creative elements" or "minor creative elements" to form we want pictures, as Figure 3 shows. The main creative elements of head can be used to combine with the minor creative elements of hat, shadow, body, and backgroup to from the Q-version comic system[11]. For that, we have provided an example of Michael Jackson and associated main creative elements and minor creative elements, as Figure 4 shows.

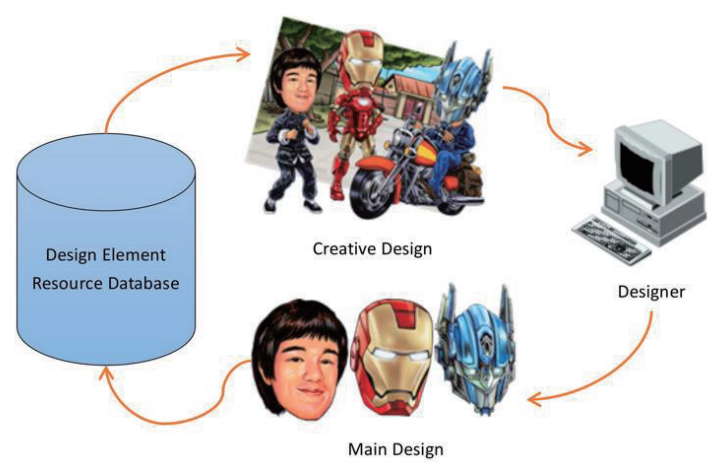

Figure 2. Online Style Database

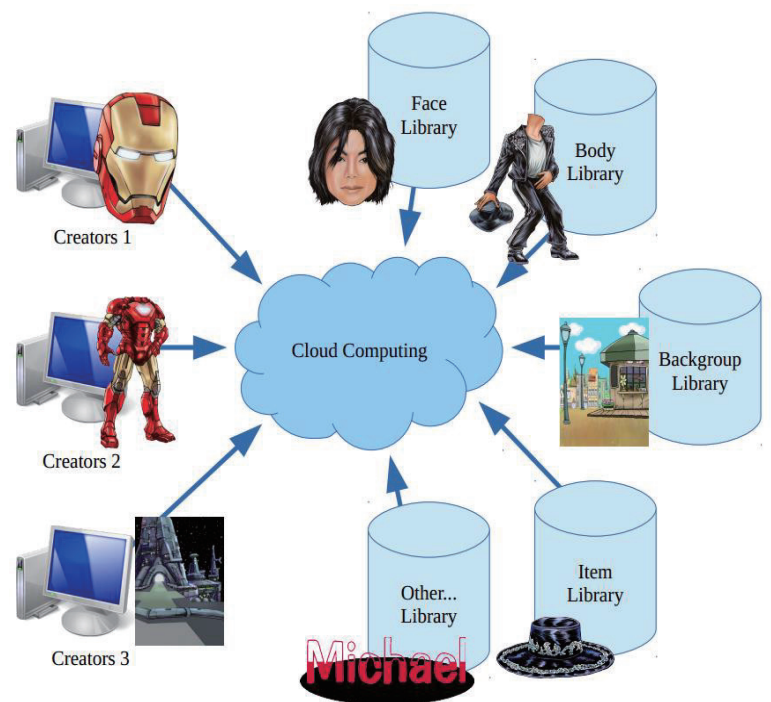

Figure 1. Cloud-based Shared Authoring System Design Architecture

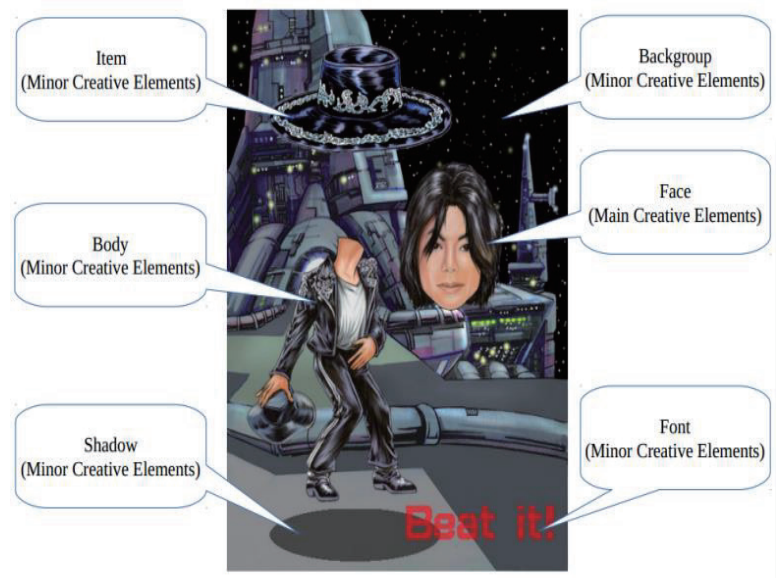

Figure 3. Analysis of Creative Elements 


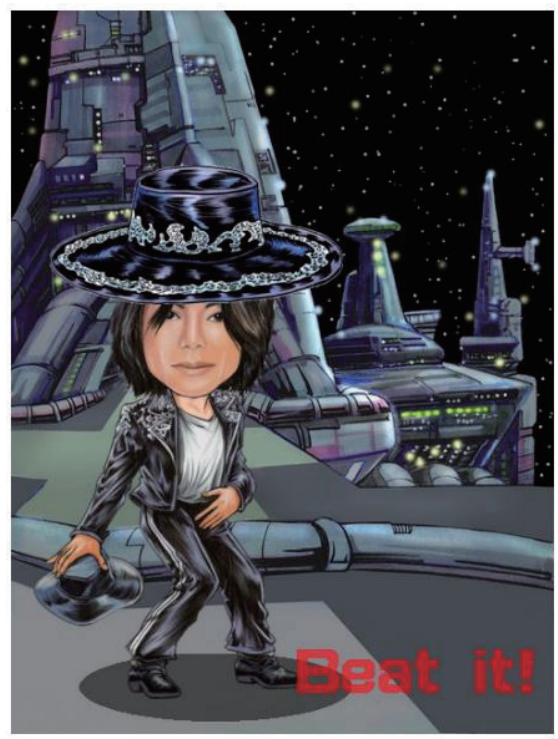

Figure 4. Q-Version Modeling System Generates Comic

\section{Methodologies Review}

In this study, we first designed to build cloud infrastructure environments, which is called "Characters Database", and in accordance with the needs of the user role modeling "Purpose Orientation" to "Conducting Greg Relation Analysis". The process "Conducting Greg Relation Analysis" is used to build the data set. The results of the analysis, create user-specific information on individual data, and these data with Rough Set algorithms to help users identify the role of modeling elements needed to help users reference to the design. Figure 5 shows the system processes to find design elements.

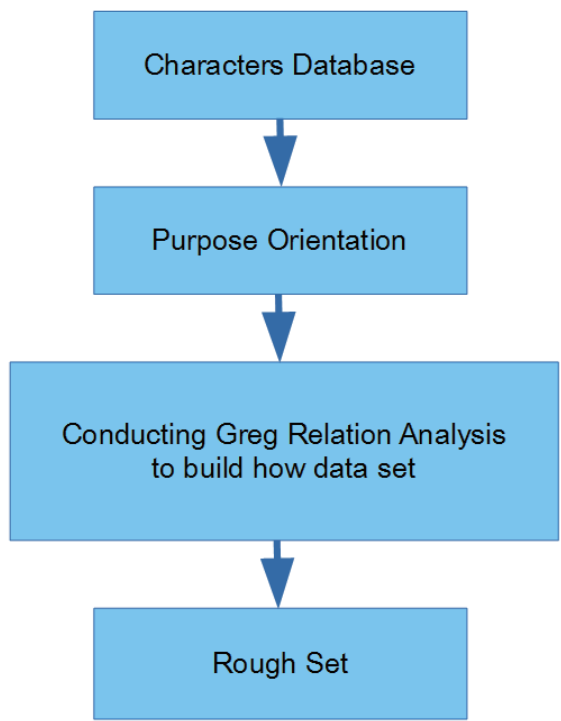

Figure 5. System processes to find design elements.

4. Methodologies And Implementation Of OSG Systems
According to online style database, we create 8,000 creative elements. From there, we use data mining technics to calculate and compare specific elements in order to create proper character style in a certain context. The online style database is defined in Table 1 for modeling database structure and Table 2 for design elements attribute structure, and the procedure of data mining examples of OSG systems output for the System implementation and demonstration examples is presented as Table 3 .

Table 1. Modeling database structure

\begin{tabular}{|l|l|}
\hline PicNumber & Modeling Number \\
\hline PicType & File Type: gif,jpg, png \\
\hline PicGroup & $\begin{array}{l}\text { Modeling Groups: head, body, } \\
\text { objects }\end{array}$ \\
\hline PicName & Style Name \\
\hline PicLevel & Modeling Rating \\
\hline PicPrice & Style Points \\
\hline PicNote & Modeling Note \\
\hline
\end{tabular}

Table 2. Design elements attribute structure

\begin{tabular}{|c|c|}
\hline PicMode & Attributes: $1=$ gif $/ 2=j p g / 3=p n g$ \\
\hline PicNumber & Picture Number \\
\hline PicSize & Picture Size: $(0 \sim 100)$ \\
\hline PicX & X Coordinate \\
\hline PicY & Y Coordinate \\
\hline PicLimpid & Transparency \\
\hline PicFlop & Opposite: $(0-1)$ \\
\hline PicRotate & Rotation Angle \\
\hline PicRGB & RGB Primary Colors \\
\hline
\end{tabular}

Table 3. System implementation and demonstration examples.

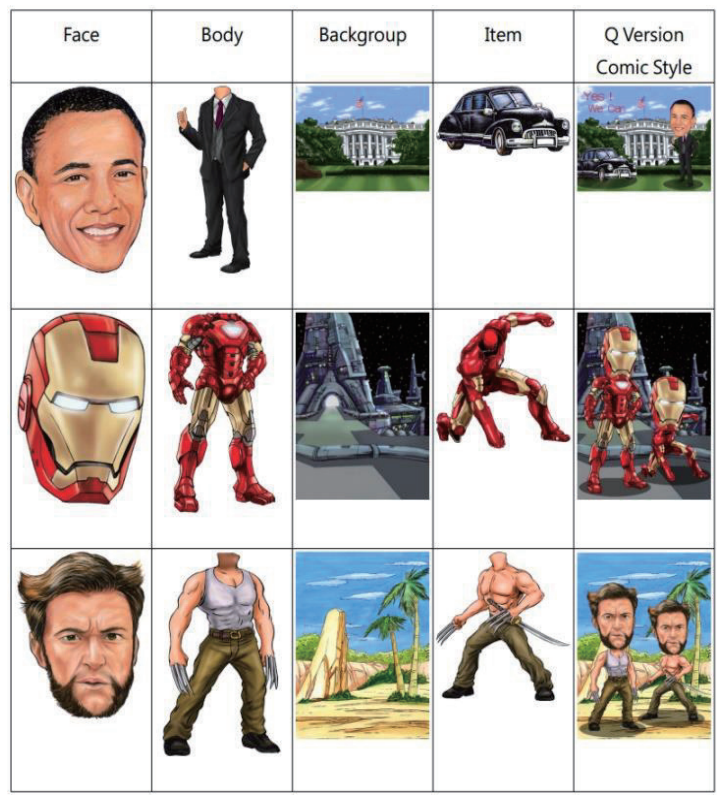

5. Conclusion 
In this study, we provide an online platform based on cloud computing which enable comic designer to design comic character more easily and efficiently. By using online Style database, online Style generating systems can help designer with different combination of character style in a certain context. Every designer who joined this program can also contribute their product to the online style database so the creative task will be much easier. The output of Q-version comic character can be used in popular online messenger such as "Line" or "WeChat", and contribute to Internet society.

\section{References}

[1] E. Davies. The script as mediating artifact in professional theater production. Archival Science 8(3), pp. 181-198. 2008.

[2] V. W. Michael, R. B. Stone, H. Thevenot and T. Simpson. Examination of platform and differentiating elements in product family de-sign. J. Intell. Manuf. 18(1), pp. 77. 2007.

[3] M. Goldner and K. Birch. Resource sharing in a cloud computing age. Interlending \& Document Supply 40(1), pp. 4-11.2012..

[4] Anonymous Pixton comics inc.; "collaborative comic creation" in patent application approval process. Telecommunications Weekly pp. 184. 2014.

[5] G. J. Shilba and S. A. Kiruba. IMPROVING PERSONALIZED IMAGE RETRIEVAL FROM THE PHOTO SHARING SOCIAL NET-WORKS. International Journal of Marketing and Technology 3(6), pp. 32-45. 2013.

[6] Tsai, S. T. (2016, November). Creative design resource integration model: Comic design element for collaborative sharing education. In Advanced Materials for Science and Engineering (ICAMSE), International Conference on (pp. 390-393). IEEE.

[7] Paracha. S, Mohammad. M.H, Khan. M.T.A, Mehmood. A and Yoshie. O. Balancing fabula \& interactivity: An approach for narrative creation in interactive virtual drama. Emerging Technologies, 2008. ICET 2008. 4th International Conference on, pp. 321-326. 2008.

[8] Junjun Qu; Li Deng; Haikuan Wang; Wenqiang Yang; Minrui Fei, Online prediction and schedule generating system based on discrete event simulation model, Control Conference (CCC), 2013 32nd Chinese, 26-28 July 2013.

[9] Sean Carlin and Kevin Curran, Cloud Computing Technologies, International Journal of Cloud Computing and Services Science (IJ-CLOSER) Vol.1, No.2, June 2012, pp. 59 65.

[10] [4] Tsai, S. T., Chang, T. C., \& Huang, Y. F. (2016). An intelligent recommendation system for animation scriptwriters' education.Eurasia Journal of Mathematics Science \& Technology Education, 12.

[11] [13] Tsai, S. T. (2017, May). Intelligent comic make system based on comic script creation. In Applied System Innovation (ICASI), 2017 International Conference on (pp. 1729-1732). IEEE.

[12] Tobita, H. (2015, November). Comic-Crowd: interactive comic creation that supports multiple storylines, visualizations, and platforms. In Proceedings of the 14th International Conference on Mobile and Ubiquitous Multimedia (pp. 163-172). ACM. 